\title{
Frequency-selective detection at three signal amplitudes
}

\author{
GORDON Z. GREENBERG ${ }^{2}$
}

UNIVERSITY OF ILLINOIS

\begin{abstract}
Using the probe-signal method, frequency-response characteristics were obtained for four Os detecting signals nominally of only $1000 \mathrm{~Hz}$ under three signal-amplitude conditions: $E / N_{o}=10.0,12.6$, and 15.8. Detection of 1000-Hz signals decreased systematically with signal-amplitude decreases, but signals at $850 \mathrm{~Hz}$ and at $1150 \mathrm{~Hz}$ were detected at or near the chance level during all signal-amplitude conditions. From the detection performance data, the detectability was inferred for four probe-signal frequencies relative to that of $1000-\mathrm{Hz}$ signals for each of the amplitude conditions. From the curves of relative detectability, half-detectability bandwidths were estimated. Neither the relative detectability curves nor the half-detectability bandwidths indicate any systematic change in the frequencyselective behavior of the auditory $O$ across the signal amplitudes used.
\end{abstract}

In many signal-detection experiments, the O's task is to detect simply the presence of signals of a single frequency presented against a background of wideband noise. An $\mathrm{O}$ listening under such conditions conceivably could ease his task by selecting from the auditory stimulus that portion of the energy best representing the signal and attenuating the rest. He might thereby enhance his signal detection by removing interference from the extraneous energy. The $\mathbf{O}$ might select by inserting a narrow-bandpass filter, tuned to the frequency of the signal, into the system delivering his input. In fact, a filter has been used in various models of the behavior of auditory $O s$ in this and similar situations (e.g., Green \& Swets, 1966; Henning, 1967; Jeffress, 1966; Schafer et al, 1950; Sherwin et al, 1956; Swets, Green, \& Tanner, 1962).

One version of such a model, which we call a sensory-filter model, would propose that the auditory system selects from the input by differentially attenuating the various frequency components during early stages of input processing. This hypothetical selection process would alter the distribution of energy in the stimulus. Input energy at the frequency of the signal would be unaffected by the process, but at other frequencies the input energy would be attenuated. The frequency-response characteristic of the sensory filter, showing the relative attenuation as a function of frequency, would be similar to that of a single-tuned, bandpass filter.

The probe-signal method has previously been used to obtain concurrent estimates of the detectabilities of signals of various frequencies from Os detecting signals of nominally a single frequency, $1000 \mathrm{~Hz}$ (Greenberg \& Larkin, 1968). From those experiments, the Os' frequency-response characteristics showed systematically differential responding as a function of the frequency of the signal, quite reminiscent of the frequencyresponse characteristics of single-tuned, bandpass filter devices. Detection was $75 \%$ to $90 \%$ correct for the $1000-\mathrm{Hz}$ "signal-to-be-detected" while the concurrent performance at $850 \mathrm{~Hz}$ and at $1150 \mathrm{~Hz}$ was at or near the chance level of $50 \%$ correct. Those results leave no doubt that the auditory $O$ in that listening situation does select from the stimulus input and respond differently as a function of the signal frequency.

In terms of a sensory-filter model, these results would indicate that signals with frequencies at or beyond $850 \mathrm{~Hz}$ and $1150 \mathrm{~Hz}$ were so severely attenuated by the filter that they were effectively removed from the input that formed the basis for the detection decisions; that is, signals at those outlying frequencies were "not heard." However, whether or not the results of those experiments represent a process adequately represented by a sensory-filter model remains to be determined.

In the report of those experiments, any claim that the results supported a sensory-filter model for the selective process was deferred until the process reflected by the probe-signal method was more fully specified. For example, are the characteristics of that process fixed or adaptable and under what auditory conditions? Swets, Green, and Tanner (1962) and Swets (1963) suggest that the parameters of the auditory system are not fixed and that frequency selection may be subject to modification to suit the O's task.

This author knows of no sensory-filter model that explicitly predicts an alteration of the $O$ 's frequency-selective process in response to a change of signal amplitude. The study reported here was conducted to investigate the stability of the $O$ 's selection process over a range of signal amplitudes. The use of the probe-signal method in this investigation will further specify the frequency-selective process being measured by that method.

\section{Method}

\section{METHOD AND PROCEDURE}

The rationale for the probe-signal method has been presented (Greenberg \& Larkin, 1968). The essential feature of the method is the evaluation of the O's listening strategy by occasionally inserting stimuli that are novel to the situation. The $O$ performs a listening task that nominally requires the detection of signals of a single frequency; these we call the primary signals. On infrequent, randomly determined trials, a probe signal is presented in lieu of the primary signal. Probe signals are presented with various frequencies sampled from a band of frequencies centered on that of the primary signal. The various probe signals and the primary signal, each of a different frequency, are all presented with equally effective energy. With estimates of the detectability of the signals of the various frequencies obtained concurrently, the frequency-response characteristic of the $\mathbf{O}$ can be specified.

\section{Procedure}

A single experimental session of 13 blocks of trials, requiring approximately $2 \frac{1}{2} \mathrm{~h}$, was conducted on any one day. A block of 85 trials lasted approximately $7 \mathrm{~min}$. A pause of 2 to $3 \mathrm{~min}$ was provided between all blocks except that twice in a session, these pauses were extended to approximately $10 \mathrm{~min}$.

The trial structure was basically that of the common, two-alternative, temporal-forced-choice experiment in which the a priori presentation probability of the signal was 0.50 for each of two observation intervals. For each trial, three 0.35 -sec intervals, the warning interval at the beginning of the trial, and the two observation intervals were defined for the $O$ by three separate indicator lamps. The first observation interval began $0.5 \mathrm{sec}$ after the termination of the warning interval and the second observation interval began $0.35 \mathrm{sec}$ after the end of the first observation interval. The response interval of $2.75 \mathrm{sec}$ began at the end of the second observation interval and continued until the onset of the warning interval of the next trial. During the warning interval of each trial a sample signal of $1000 \mathrm{~Hz}$ was presented. The duration and amplitude of the $1000-\mathrm{Hz}$ sample 
signal was the same as that of the $1000-\mathrm{Hz}$ signal during the observation interval of a trial. All signals occurred during the latter $0.25 \mathrm{sec}$ of a 0.35 -sec interval.

Certain aspects of the experimental procedure were intended to be known by the Os but others were not. In the following, the procedural details presented first are those that were to describe the experimental situation for the Os. Then, the additional procedures that were followed will be described to specify completely the experimental situation.

The initial instructions given the Os were written as though designed for an experiment to investigate the detection of signals of a single frequency. The foregoing details of the trial structure were explained to the Os. The instructions included attempts to encourage the Os to believe that the frequency of all the signals would be the same, but omitted any explicit statement to that effect. The Os were to indicate by their responses only the presence of the signal during one of the observation intervals of each trial. The Os received both initial information that three signal amplitudes would be presented and information about the particular signal amplitude each time it was changed during the experiment.

To reorient the Os during the first trial block of each session, $1000-\mathrm{Hz}$ signals were presented and each response was followed by feedback to each $O$ indicating whether his response had been correct or incorrect. The feedback was provided by indicator lamps illuminated at the end of the response and extinguished at the beginning of the warning interval of the next trial. No further feedback was provided after the first block of a session.

The remaining 12 blocks of each session were divided into three groups of four consecutive blocks. During each of the three groups of blocks, $1000-\mathrm{Hz}$ signals were presented at one amplitude so that $\mathrm{E} / \mathrm{N}_{\mathrm{o}}$ equaled $10.0,12.6$, or 15.8 . These values represent successive increments of $1 \mathrm{~dB}$ in the signal amplitude. The order of presentation of those signal amplitudes within a session was counterbalanced over every 6 of the 18 sessions of the experiment. The amplitude of $1000-\mathrm{Hz}$ signals during Block 1 of a session was the same as during Blocks 2 through 5 of that session.

Those aspects of the procedure in the foregoing account that were meaningful to the Os were made obvious by the instructions. The Os were informed of the type of trial that presented a signal of $1000 \mathrm{~Hz}$ during one of the observation intervals, denoted here as a primary-signal trial. In fact, another type of trial was also presented but the Os were not explicitly informed of this. During this second type of trial, a probe-signal trial, the signal presented during one of the observation intervals, a probe signal, was of a frequency other than $1000 \mathrm{~Hz}$. The two types of trials differed only in the specifications of the signals presented during the observation intervals.

Probe-signal trials were not presented during the first trial block of a session. During the remaining 12 blocks of each session, probe-signal trials as well as primary-signal trials were presented. Probe signals of $850,925,975,1025,1075$, and $1150 \mathrm{~Hz}$ were used. The probe signals during any one block of trials were all of the same frequency. Four of the six probe-signal frequencies were presented during a given session. Probe signals of each of those four frequencies were presented during each of the three amplitude conditions for the primary signals. The order of presentation of the four probe-signal frequencies within a group of blocks with the same primary-signal amplitude was different for each such group of blocks within a session. The sequence of probe-signal frequencies over sessions was arranged so that each combination of probe-signal frequency and amplitude was presented during two blocks every three sessions.

In blocks with both types of trials, the order of presentation of the two types of trials was determined by a table of random numbers (Rand Corporation, 1955). On any given trial, the type of trial presented was instrumentally independent of the observation interval in which the signal was presented and these two events were determined by separate, consecutive sampling from the same random number source. The nominal (sampling) presentation probability was $\mathbf{0 . 6 0}$ for the primary-signal trial and 0.40 for the probe-signal trial, but a further constraint was imposed on the trial sequence so that two probe-signal trials could not occur consecutively in a block of trials. Probe signals were observed to occur on approximately $28 \%$ of the trials.

For a given amplitude of the primary signal, probe signals at the various frequencies were presented with levels chosen in an attempt to produce equal detectability for all the probe signals and the primary signal. We therefore say for a given amplitude condition that all signals were presented with "equally effective energy." The amplitude of each probe signal was adjusted in proportion to the separation of its frequency from the frequency of the primary signal (Green, McKey, \& Licklider, 1959). Specifically, probe signals with frequencies below that of the primary signal were presented with amplitudes lower than that of the primary signal by $0.2 \mathrm{~dB}$ per $100-\mathrm{Hz}$ separation from the frequency of the primary signal. The amplitude of each probe signal with a frequency higher than that of the primary signal was determined by the same relation and was greater than that of the primary signal.

For each O, the earphones (Permoflux PDR-10) of a binaural headset were wired in parallel and in phase. Several headsets were wired in parallel. A noise generator (Grason-Stadler Model 455-C) provided a continuous noise with an overall level of approximately $65 \mathrm{~dB}$ SPL. The 0.25 -sec signal was added to the noise by gating an oscillator output without regard to phase and without using special devices.

In an attempt to prevent a gradual, overall performance decline, a monetary incentive was introduced during the second half of the experiment. For each $O$, his mean performance level on primary signals during the first half of the experiment determined a criterion level of performance for the second half. For each session of the second half in which his performance attained the criterion level, he earned a monetary bonus in addition to his normal hourly rate of pay. Each $\mathrm{O}$ was informed of his criterion level of performance and he was given a report each session of his performance during the previous session.

Three young women and three young men, all with clinically normal hearing, served as simultaneous Os for five familiarization sessions and for the following 18 experimental sessions. These Os had no previous experience in auditory experiments.

\section{RESULTS AND DISCUSSION}

The data are not presented for certain Os, sessions, and blocks of trials for the following reasons: two Os were excessively absent from the experimental sessions; an apparatus malfunction occurred during one session; and the first trial block of each session was presented only to reorient the Os. Performance levels were not appreciably different before and after the monetary incentive for performance was introduced, and the data from both groups of sessions were combined.

In the two-alternative, forced-choice procedure, an $\mathrm{O}$ may prefer a given response independent of the actual locations of the signals. The amplitude of the signal may determine the degree to which such response preference is represented in the performance data. To enable comparisons of the performance levels for the various signal amplitudes the obtained per cent correct, $p(C)$, data were adjusted for response preference in accordance with the procedure presented by Green and Swets (1966). From the data for each combination of frequency and amplitude for each $\mathrm{O}$, estimates were obtained of the conditional probabilities of an 


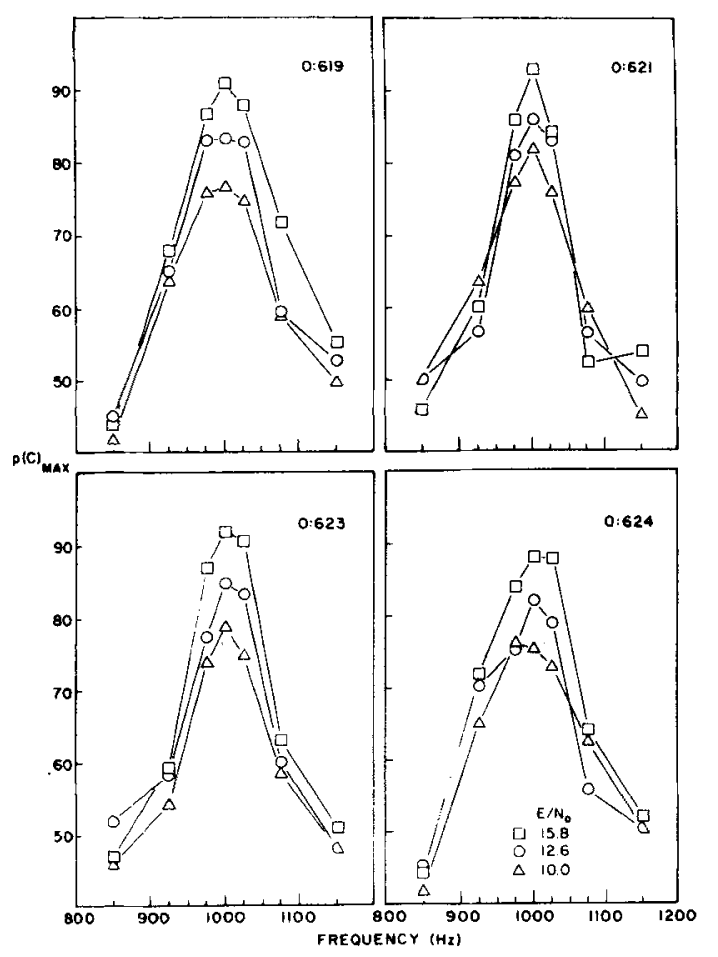

Fig. 1. Frequency-response characteristics of four Os for three signal-amplitude $\left(E / N_{0}\right)$ conditions. Each curve shows the mean detection performance corrected for response preference, $\mathrm{p}(\mathrm{C})_{\mathrm{MAX}}$, for signals of seven different frequencies all presented with "equally effective energy." $A \mathrm{p}(\mathrm{C})_{\mathrm{MAX}}$ of 50 represents performance at the chance level.

Interval-1 response given an Interval-1 signal presentation, and of an Interval-1 response given an Interval-2 signal presentation. With those two probability estimates a $d^{\prime}$ for each frequencyamplitude combination was obtained from a table for the normal curve. This $\mathrm{d}^{\prime}$ provided a basis for the computation of $\mathrm{d}_{\mathrm{M}}^{\prime} \mathrm{AX}$ and of $\mathrm{p}(\mathrm{C})_{\mathrm{MAX}}$. Each of the $\mathrm{p}(\mathrm{C})_{\mathrm{MAX}}$ values was within $3 \%$ of the value of the corresponding $\mathrm{p}(\mathrm{C})$ before adjustment.

Figure 1 shows the results for four Os. Each of the three curves for a given $O$ represents estimates derived from the data for one of the three amplitude conditions. For each curve, the data point at $1000 \mathrm{~Hz}$ is based on approximately 4100 primary-signal trials during 17 sessions. The data points for each of the probe-signal frequencies at each signal level are based on approximately 270 trials during 11 or 12 sessions. The curves show that signals of $850 \mathrm{~Hz}$ and of $1150 \mathrm{~Hz}$ were detected at or near the chance level while the concurrent performance at $1000 \mathrm{~Hz}$ was $75 \%$ to $93 \%$ correct. The general shape of the curves is quite similar to that obtained in other experiments (Greenberg \& Larkin, 1968), and it is suggestive of frequency-response characteristics of bandpass filter devices.

The changes in signal amplitude used in this experiment should have had effects on signal detectability comparable to those obtained in other experiments. For the primary signals of $1000 \mathrm{~Hz}$, the change in detectability as a function of signal power $\left(E / N_{0}\right)$ is plotted for each $O$ in Fig. 2 where the detectability measure $d^{\prime}$ MAX was derived from the per cent correct data represented by the data points at $1000 \mathrm{~Hz}$ in Fig. 1. The solid line inset into Fig. 2 indicates the slope of the line representing a change in $\mathrm{d}_{\mathrm{MAX}}^{\prime}$ by a factor of 2 for a change in signal power or $E / N_{0}$ by a factor of 2 (i.e., a 3-dB change in signal power). For

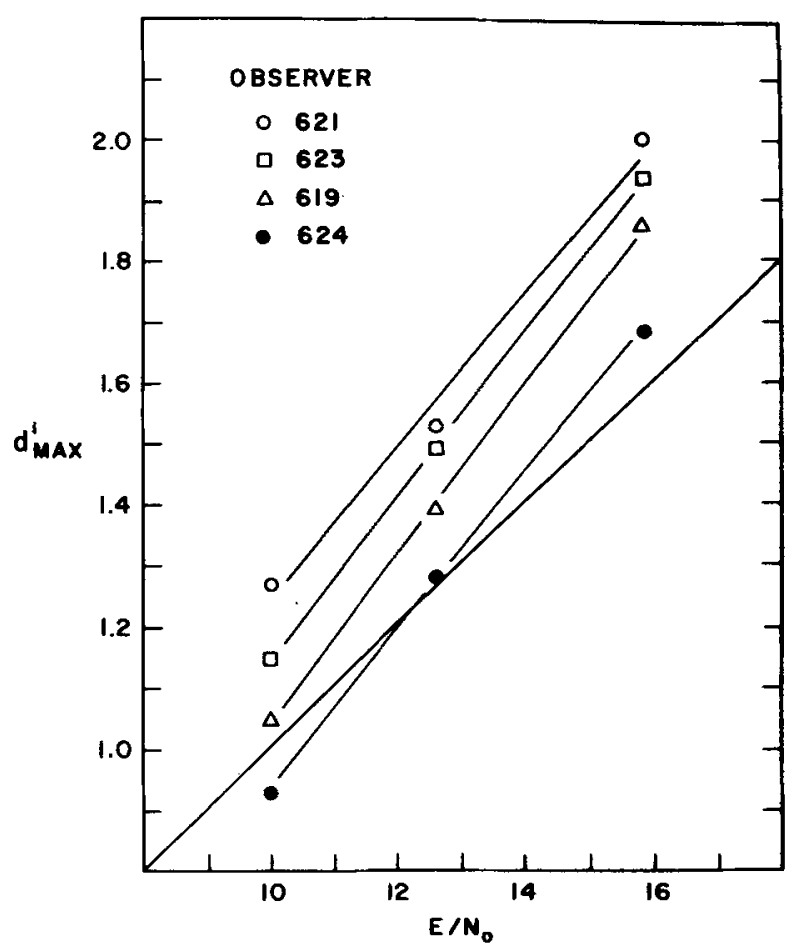

Fig. 2. Psychometric functions of four Os for $1000-\mathrm{Hz}$ signals at three signal amplitudes $\left(E / N_{0}\right)$. The detectability measure $d^{\prime} M \dot{A X}$ was derived from the $1000-\mathrm{Hz}$ performance data of Fig. 1. The inset solid line indicates a relation producing a change in detectability by a factor of 2 for a change by a factor of 2 in signal power, $3 \mathrm{~dB}$.

the 2-dB change in signal power used in this experiment, that relation would produce a change in $\mathrm{d}^{\prime} \mathrm{MAX}$ by a factor of 1.58 . The slopes of the obtained psychometric functions are somewhat greater than that of the inset solid line. The linearity as well as the slopes of those curves relating detectability and signal power are consistent with other psychometric functions obtained under comparable conditions (e.g., Green \& Swets, 1966). The curves of Fig. 2 do not indicate that the presence of the probe signals in this experiment altered the expected response of auditory Os to the changes in signal amplitude used.

To relate the results of the experiment to the stability of the $O$ 's selection process, three aspects of the data were examined: (1) The detection performance shown in Fig. 1. (2) The inferred relative detectability; from the differential detection of the signals shown in Fig. 1 , the detectability of different signals relative to that of the $1000-\mathrm{Hz}$ signals was inferred. The constancy of that relative detectability across the three signalamplitude conditions was examined. (3) The half-detectability bandwidths were measured from the curves of relative detectability.

\section{Detection Performance}

In Fig. 1, each of the detection performance curves for a given amplitude condition shows differential detection as a function of the signal frequency. For at least some of the signal frequencies the detection of signals of a given frequency changed as a function of the signal amplitude. However, it is difficult to conclude much concerning the stability of the $O$ 's selection process from a comparison of the three detection performance curves for a given $O$ for the following reasons. 


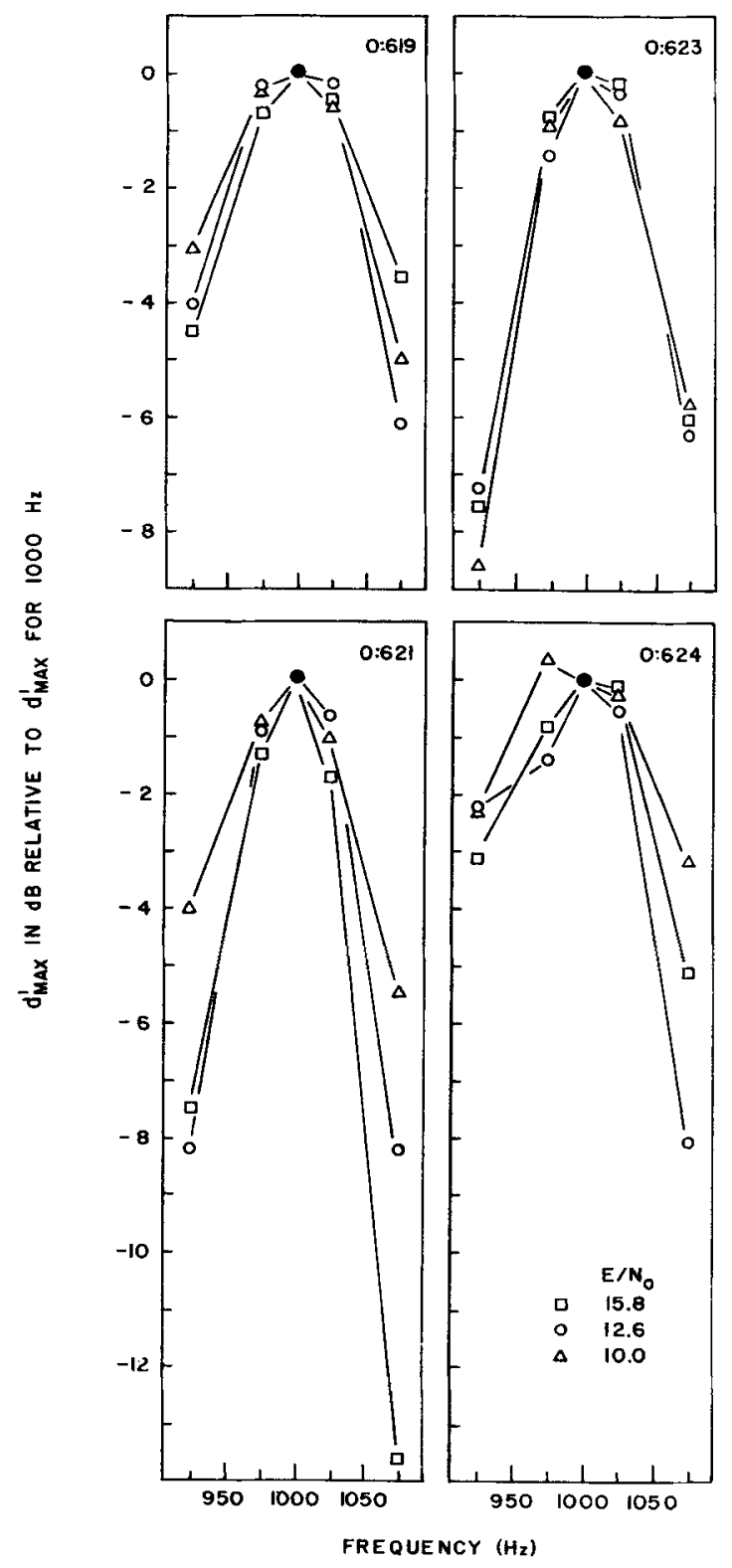

Fig. 3. The detectability of probe signals relative to that of $1000-\mathrm{Hz}$ signals of comparable amplitude expressed in decibels for each of the three amplitude conditions and for four Os. Half-detectability bandwidths were determined at the $-3-\mathrm{dB}$ points of these curves.

Assuming that the $O$ 's selection process differentially attenuates signals as a function of frequency, like a filter, then the stability of that process would not be immediately obvious in a comparison of the detection performance curves because $\mathrm{p}(\mathrm{C})_{\mathrm{MAX}}$ is not linearly related to signal energy. For that reason, the differences and similarities among the three curves cannot be interpreted directly in terms of amounts of attenuation of signal energy. Although the lack of any systematic alteration in the detection of signals of $850 \mathrm{~Hz}$ and of $1150 \mathrm{~Hz}$ is perhaps a feature of the curves in Fig. 1 indicative of stability, only certain extreme alterations in the attenuation by the $O$ 's selection process would be reflected clearly in comparisons of the entire curves of detection performance. Rather, the stability of a filter-like selection process would be indicated by the stability of the differential attenuation or some linearly related measure across the amplitude conditions.

The detectability measure $d^{\prime}$ has been shown to be linearly related to signal energy, at least over the range of signal amplitudes used in this experiment (Green \& Swets, 1966) and Fig. 2 shows this relation held, approximately, for this experiment. The differential attenuation by the hypothetical sensory-filter, producing different effective energies as a function of signal frequency, would be directly reflected in different $d^{\prime}$ values. If a curve of detectability as a function of frequency is obtained for each amplitude condition a comparison can be made of the effective energy at each frequency across the three amplitude conditions.

For a filter, a plot of the attenuation of various frequencies relative to that of the frequency at the center of the passband is commonly used as a frequency-response curve (Beranek, 1949). In a similar manner, a curve of the detectabilities of the probe signals relative to that of the $1000-\mathrm{Hz}$ signals would reflect the attenuation effected by the $O$ 's selection process. From the differential detection performance data for a given amplitude condition in Fig. 1, differential detectabilities may be inferred for the various probe signals and related to the detectability of the $1000-\mathrm{Hz}$ signals to obtain relative-detectability curves. From a filter, the same relative attenuation curve across the three signal-amplitude conditions of this experiment would indicate the filter had remained stable. Then, similar relative-detectability curves across amplitude conditions would imply stability of the O's selection process.

\section{Inferred Relative Detectability}

To obtain relative-detectability curves, the detectability measures were treated similarly to measures of the output of a filter. For each probe signal at each signal level a ratio was formed of the $d^{\prime}{ }_{M A X}$ for that frequency to the $d^{\prime}{ }_{M A X}$ for the $1000-\mathrm{Hz}$ signals at that amplitude. Most of the relative $d_{M}^{\prime}{ }_{M X}$ values derived in that manner for the $850-\mathrm{Hz}$ and for the $1150-\mathrm{Hz}$ signals were extremely small or indeterminate. The detection performance data of Fig. 1 shows no systematic changes at those frequencies, so the data for those probe signals were excluded from further transformation.

In Fig. 3 the relative $d^{\prime}{ }_{M A X}$ values are plotted for five of the frequencies presented at each of the three signal levels. The values of the ratios of the detectability of each probe-signal frequency to that of the $1000-\mathrm{Hz}$ signals at a comparable signal amplitude are expressed in decibels on the ordinate. To compare the three curves for a given $O$, the relation between detectability and signal power is assumed to be linear, implying that a given amount of attenuation of the signal power by the frequency-selective process produced a given value of relative detectability.

In general, the changes in relative detectability across the signal-amplitude $\left(E / N_{0}\right)$ conditions were most marked for the 925-Hz and $1075-\mathrm{Hz}$ signals. The curves for $\mathrm{O} 621$ show relatively sizable and orderly decreases in relative detectability in response to the decrease in signal level. For some of the other Os the curves show some similar changes at $925 \mathrm{~Hz}$ and/or at $1075 \mathrm{~Hz}$ but there is no obvious systematic trend. Perhaps another aspect of the relative detectability curves would be more indicative of the state of the $O$ 's selection process under the three amplitude conditions.

\section{Half-Detectability Bandwidths}

One common specification of the frequency-response characteristic of a bandpass filter is by its half-power bandwidth (Lawson \& Uhlenbeck, 1950). For a bandpass filter device centered on $1000 \mathrm{~Hz}$, an alteration of its frequency-response 
Table 1

Performance on $1000 \mathrm{~Hz}$ Signals and Half-Detectability Bandwidths

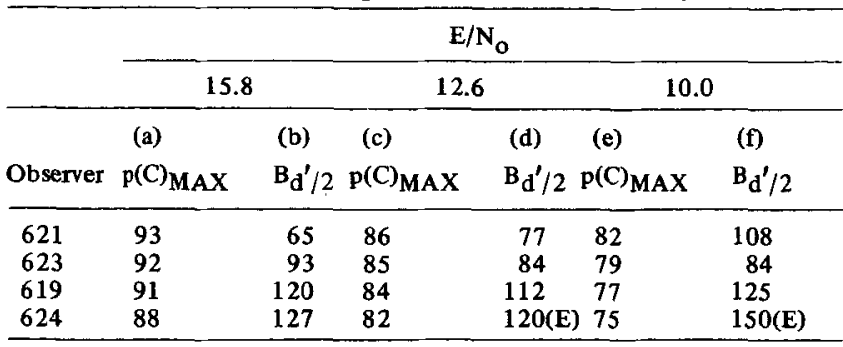

characteristic very likely would be reflected by changes in its half-power bandwidths. In the context of a sensory-filter model, each curve of Fig. 3 is tantamount to a frequency-response characteristic of an $O$ 's selection process. Each of those curves were measured to obtain the half-detectability bandwidths, measures analogous to the half-power bandwidth of a filter device.

For a filter device the half-power bandwidth is the width of the filter's passband between the frequencies at which it attenuates signals to a level of one-half the power passed at the center frequency of the passband. The half-power bandwidth of a filter is then the width between the frequencies which are attenuated by $3 \mathrm{~dB}$.

The half-detectability points on the relative-detectability curves are defined as those where the relative detectability was $-3 \mathrm{~dB}$. The half-detectability bandwidths, $\mathbf{B}_{\mathrm{d}^{\prime} / 2}$, visually determined from the curves of Fig. 3 , are given in Table 1 (Columns b, $d$, and $f$ ). The bandwidth estimates (in hertz) are fairly crude because of the number and location of the data points determining a curve and for $O 624$, the bandwidths had to be estimated (E) for the two lower amplitude concitions using extrapolations of the curves. In conjunction with the changes in signal amplitude $\left(\mathrm{E} / \mathrm{N}_{0}\right)$ there is no discernable trend common across Os. Only the data for $\mathrm{O} 621$ approximates an orderly change in bandwidth accompanying the decrease in signal amplitude, and that result is questionable because, of the four psychometric functions in Fig. 2, the deviation from linearity was greatest for $\mathrm{O}$ 621. The half-power bandwidth is only one of several ways of specifying the frequency-response characteristic of a filter (e.g., Swets, Green, \& Tanner, 1962). Consideration of additional measures of this type does not appear appropriate in view of the precision of the estimates possible from the data.

One aspect of the data, although unaccountable, may warrant note. The presentation of the data in Table 1 has the Os ordered according to their detection performance on the $1000-\mathrm{Hz}$ signal at the highest signal level (Column a). The same ordering of performance across $O$ s holds at the other signal levels (Columns $\mathrm{c}$ and e). That order is shown in Fig. 2. For each of the three signal levels, the half-detectability bandwidth estimates, $B_{\mathbf{d}^{\prime} / 2}$, are likewise ordered across $O$ s with the exception of those for Os 621 and 623 for the lowest signal level. A comparison of the curves of Fig. 1 discloses that relation among Os. As in the results of previous experiments (Greenberg \& Larkin, 1968), here also there is some suggestion that across Os the smaller bandwidth estimates are associated with higher detection performance on the primary signal.

\section{SUMMARY AND CONCLUSION}

Curves of detection performance for signals at three amplitudes all show systematic differences in the detectabilities of signals of different frequencies. With two successive 1-dB decreases of the signal amplitude, the detection of $1000-\mathrm{Hz}$ signals systematically decreased, but performance on the probe signals of $850 \mathrm{~Hz}$ and of $1150 \mathrm{~Hz}$ remained at or near the chance level.

The detection performance data were evaluated for evidence of changes in some process like a sensory filter underlying the frequency-selective behavior of the Os. The data were treated similarly to the measures of the output of a filter device. The detectabilities of various frequencies relative to that of $1000 \mathrm{~Hz}$ were inferred from the performance data. From the curves of relative detectability, half-detectability bandwidths were measured. If the frequency-selective process were stable over the signal amplitudes used, the relative detectability curves would remain stable and/or the half-detectability bandwidths would remain constant.

The data do not indicate any systematic changes in the $O$ 's selection process in response to the changes in signal amplitude used. The results of this experiment are not discrepant with a sensory-filter model for the $O$ 's frequency-selective auditory behavior. The results further support the utility of the probe signal method for exploring the frequency-selective behavior of the auditory $O$.

\section{REFERENCES}

BERANEK, L. L. Acoustic measurements. New York: Wiley, 1949.

GREEN, D. M., BIRDSALL, T. G., \& TANNER, W. P., JR. Signal detection as a function of signal intensity and duration. Journal of the Acoustical Society of America, 1957, 29, 523-531.

GREEN, D. M., MCKEY, M. J., \& LICKLIDER, J. C. R. Detection of a pulsed sinusoid in noise as a function of frequency. Journal of the Acoustical Society of America, 1959, 31, 1446-1452.

GREEN, D. M., \& SWETS, J. A. Signal detection theory and psychophysics. New York: Wiley, 1966.

GREENBERG, G. Z., \& LARKIN, W. D. The frequency-response characteristic of auditory observers detecting signals of a single frequency in noise: The probe-signal method. Journal of the Acoustical Society of America, 1968, 44, 1513-1523.

HENNING, G. B. A model for auditory discrimination and detection. Journal of the Acoustical Society of America, 1967, 42, 1325-1334.

JEFFRESS, L. A. Stimulus-oriented approach to detection re-examined. Journal of the Acoustical Society of America, 1967, 41, 480-488.

LAWSON, J. L., \& UHLENBECK, G. E. Threshold signals. New York: McGraw-Hill, 1950.

RAND CORPORATION. A million random digits with 100,000 normal deviates. Glencoe. Ill.: Free Press, 1955.

SCHAFER, T. H., GALES, R. S., SHEWMAKER, C. A., \& THOMPSON, $P$. $O$. The frequency selectivity of the ear as determined by masking experiments. Journal of the Acoustical Society of America, 1950, 22, $490-496$.

SHERWIN, C. W., KODMAN, F., JR., KOVALLY, J. J., PROTHE, W. C., \& MELROSE, J. Detection of signals in noise: A comparison between the human detector and an electronic detector. Journal of the Acoustical Society of America, 1956, 28, 617-622.

SWETS, J. A. Central factors in auditory frequency selectivity. Psychological Bulletin, 1963, 60, 429-440.

SWETS, J. A. (Ed.), Signal detection and recognition by human observers. New York: Wiley, 1964.

SWETS, J. A., GREEN, D. M., \& TANNER, W. P., JR. On the width of critical bands. Joumal of the Acoustical Society of America, 1962, 34, 108-113.

\section{NOTES}

1. The research was supported by Grant NB 05636 from the National Institute of Neurological Diseases and Blindness, United States Public Health Service.

2. Address: Hearing Research Laboratory, Department of Psychology, University of Illinois, Urbana, Illinois 61801 .

(Accepted for publication April 14, 1969.) 